\section{CONTRIBUCIÓN GENÉTICA DEL CRIOLLO JALA EN VARIEDADES ELOTERAS DE MAÍZ}

\section{GENETIC CONTRIBUTION OF JALA LANDRACE TO GREEN CORN VARIETIES}

\author{
Roberto Valdivia-Bernal ${ }^{{ }^{*}}$, F. de Jesús Caro- \\ Velarde $^{1}$, Raúl Medina-Torres ${ }^{1}$, Margarito Ortiz- \\ Catón', Alejandro Espinosa-Calderón', Víctor A. \\ Vidal-Martínez ${ }^{3}$ y Alejandro Ortega-Corona ${ }^{4}$
}

\begin{abstract}
${ }^{1}$ Unidad Académica de Agricultura, Universidad Autónoma de Nayarit, km 9 Carretera Tepic-Compostela, 63780, Xalisco, Nayarit, Tel. y Fax (311) 211-1163. ${ }^{2}$ Campo Experimental Valle de México, Instituto Nacional de Investigaciones Forestales, Agrícolas y Pecuarias, km 18.5 Carr. Los Reyes-Lecherías, Chapingo, Edo. de México. ${ }^{3}$ Campo Experimental Santiago Ixcuintla, Instituto Nacional de Investigaciones Forestales, Agrícolas y Pecuarias, km 5 Carr. Entronque internacionalSantiago Ixcuintla, Nayarit. ${ }^{4}$ Campo Experimental Valle del Yaki, Instituto Nacional de Investigaciones Forestales, Agrícolas y Pecuarias, calle Dr. Norman E. Borlaug km 12 Cajeme, Son.
\end{abstract}

* Autor para correspondencia (rvb uan@hotmail.com)

\section{RESUMEN}

El maíz (Zea mays L.) criollo de la raza Jala es apreciado por su calidad para consumo en elote; sin embargo, posee características agronómicas indeseables como plantas altas, de hasta $5 \mathrm{~m}$, muy tardío y escasa adaptabilidad. Para contribuir al aprovechamiento de las características eloteras del maíz Jala en el desarrollo de variedades mejoradas, en este estudio se evaluó su aptitud combinatoria para dichas características cuando Jala es cruzado con variedades comerciales. El trabajo se efectuo en Xalisco, Nayarit, México, con seis maíces reconocidos como eloteros: tres criollos (Mecatán, Morado y Jala) y tres híbridos comerciales ('A7573', 'D880' y 'B810'). En el 2006 se aplicó un esquema de cruzamientos dialélico completo, de acuerdo con el modelo I de Griffing y la evaluación se realizó en dos fechas de siembra durante el 2007. El análisis de varianza detectó significancia $(P \leq 0.05)$ para rendimiento de elote (Rend), número total de granos (NG), número de hileras (NH) en el elote, peso de 200 granos de elote (PF200), días a floración (DF), contenido de sólidos solubles totales (SST), vida de anaquel (VA) y diámetro del elote (DE). La aptitud combinatoria general (ACG) fue significativa $(P \leq 0.05)$ en todas las variables, excepto en rendimiento. Las variables con significancia para la aptitud combinatoria específica (ACE) fueron Rend, SST, VA y DF; los efectos recíprocos fueron significativos para Rend, SST NH, PF200 y VA. Los efectos aditivos fueron más importantes que los no aditivos; por tanto, los métodos de selección recurrente serían más apropiados para el desarrollo de variedades eloteras mejoradas de polinización libre. El maíz criollo Jala puede aportar efectos genéticos favorables para el desarrollo de variedades o híbridos eloteros.
Palabras clave adicionales: Zea mays, cruzamiento dialélico, efectos aditivos, sólidos solubles totales.

\section{SUMMARY}

Native Jala maize (Zea mays L.) race has quality attributes for consumption as green corn; however, it also possess some undesirable agronomic traits such as tall and late plants and poor adaptability. To contribute in the utilization of advantageous green ear characteristics of Jala maize in development of improved varieties, its combining ability was analized for green corn characteristics when Jala is crossed with commercial varieties. The study was carried out in Xalisco, Nayarit, México using green-ear producing varieties as parents: three commercial hybrids ('A7573', 'B810' and 'D880') dialed with three maize landraces (Jala, Mecatán and Morado). All dialled combinations were obtained in 2006, following the Griffing is Method I, and were field evaluated during 2007 in two sowing dates. Analysis of variance detected significance $(P \leq \mathbf{0 . 0 5})$ on green ear yield (Rend), total number of grains (NG) per ear, number of rows in the ear $(\mathbf{N H})$, weight of 200 fresh green grains (PF200), days to silking (DF), total solid solutes content (SST), shelf-life (VA), and ear diameter (DE). General combining ability (GCA) was significant in all traits (P $\leq \mathbf{0 . 0 5}$ ), except Rend, where specific combining ability (SCA) was more relevant. Significant traits for SCA were SST, VA and DF. Reciprocal effects were significant for Rend, SST NH, PF200 and VA. Additive effects were more important than non-additive ones; therefore, recurrent selection methods are more appropriate for the development of improved green corn open-pollinated varieties. Jala maize can contribute favorable genetic effects for developing improved green corn varieties or hybrids.

Index words: Zea mays, diallelic crossing, additive effects, total solid solutes.

\section{INTRODUCCIÓN}

El maíz (Zea mays L.) de la raza Jala ha sido cultivado en el Valle de Jala, Nayarit, México, durante décadas (Aguilar et al., 2006). Su principal uso es para consumo de elotes, y también su grano maduro se usa para preparar un platillo tradicional denominado "pozole". La mazorca de este maíz (Figura 1) ha sido reconocida mundialmente como la de mayor tamaño, hasta de $60 \mathrm{~cm}$ de longitud; sin embargo, su planta es de gran altura, con más de $5 \mathrm{~m}$ (Rice et al., 2006), y muy tardía. En los últimos años ha sufrido "erosión genética", que le ha provocado reducción en su longitud de mazorca y cambio en tipo de grano y mazorca; en consecuencia, su aprovechamiento se ha reducido a tal grado que actualmente sólo se cultivan alrededor de 30 ha anuales (Aguilar et al., 2006).

En años recientes, se ha realizado investigación en la raza Jala con el propósito de recuperar el tipo original (Rice et al., 2006; Aguilar et al., 2006), pero no se conoce de investigación en la que se usa para el desarrollo de maíces mejorados específicos para elote. 


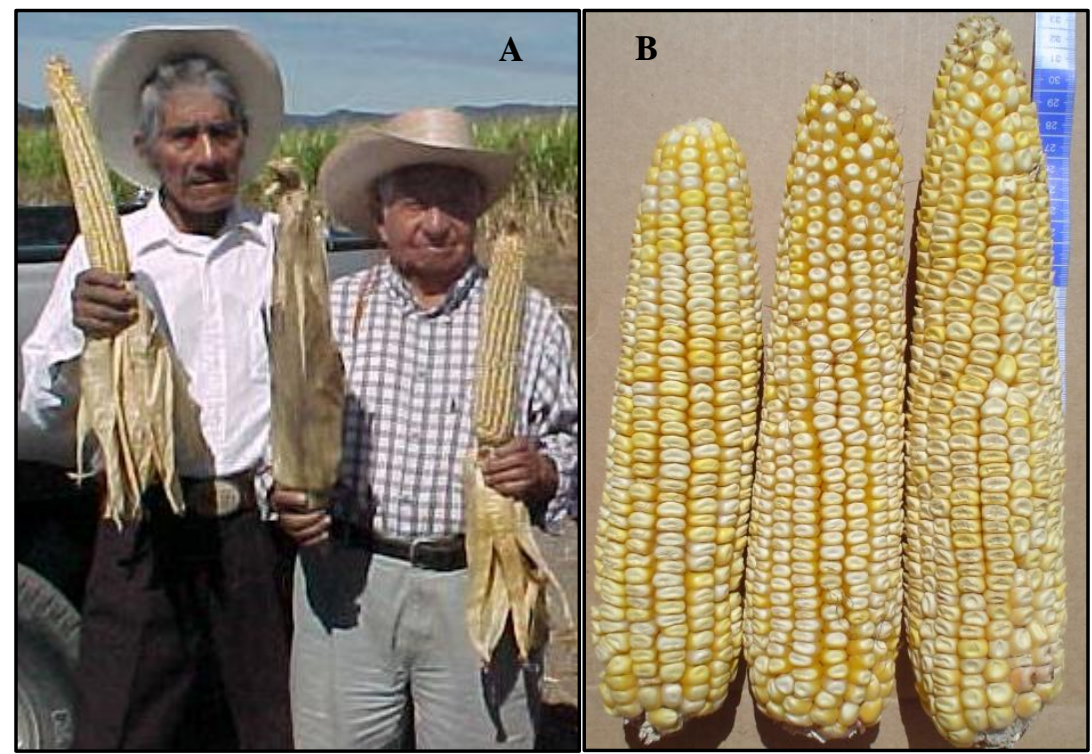

Figura 1. Mazorcas del maíz Jala producidas por agricultores del Estado de Nayarit, Mexico. (A), mazorcas experimentales (B).

Con el propósito de aprovechar su potencial genético, la variedad criolla de Jala se podría combinar con otros tipos de maíz con usos similares, bajo la hipótesis de que sus cualidades genéticas pueden recombinarse o transferirse a otros maíces de uso más común. Una primera fase de este tipo de investigación es conocer la aptitud combinatoria de sus cualidades genéticas.

El objetivo de este estudio fue estimar las aptitudes combinatorias general y específica de tres criollos de la raza Jala en cruzamientos con maíces comerciales con características de grano elotero.

\section{MATERIALES Y MÉTODOS}

El estudio se llevó a cabo en Xalisco, Nayarit, México, en el $\mathrm{km} 9$ de la carretera Tepic-Compostela, en las coordenadas $21^{\circ} 26^{\prime} \mathrm{LN}$ y $104^{\circ} 53^{\prime} \mathrm{LO}$, a una altitud de $922 \mathrm{~m}$, cuyo clima es subtropical.

Se generaron 36 tratamientos resultantes de un juego completo de cruzamientos entre seis materiales genéticos, de acuerdo con el método I de Griffing (1956), que fueron: 15 cruzas directas, 15 cruzas recíprocas y los seis progenitores (las poblaciones nativas Mecatán, Morado y Jala, y los híbridos comerciales 'A7573', 'D880' y 'B810').

Los 36 tratamientos se evaluaron en Xalisco, Nayarit, bajo condiciones de temporal o secano, en el ciclo Primavera-Verano del 2007. Se utilizó un diseño látice simple, con dos repeticiones por fecha de siembra, con una parcela experimental de cuatro surcos de $5 \mathrm{~m}$ de longitud separados a $80 \mathrm{~cm}$; los dos surcos centrales se usaron como parcela útil. Después del aclareo se dejó una planta cada $20 \mathrm{~cm}$ para obtener una densidad de población de 62500 plantas por hectárea. Se sembraron dos fechas de siembra separadas cada $15 \mathrm{~d}$ y la primera fecha fue el 15 de junio. Otras dos fechas de siembra posteriores se debieron desechar porque las plantas fueron severamente afectadas por el exceso de lluvia. Los dos experimentos (dos fechas de siembra) fueron manejados de acuerdo con las recomendaciones del Instituto Nacional de Investigaciones Forestales Agrícolas y Pecuarias (INIFAP) para Nayarit (Vidal, 1993), y se fertilizo con la fórmula $100 \mathrm{~N}-60 \mathrm{P}-20 \mathrm{~K}$.

Los datos de elote se obtuvieron de 10 elotes cosechados de 15 a 18 d después de floración femenina, de plantas con competencia completa. Cinco elotes sin hojas ni pedúnculo se usaron para medir el rendimiento y sus componentes: rendimiento de elote (Rend; $\mathrm{kg} \mathrm{ha}^{-1}$ ), longitud del elote (LE; cm), diámetro del elote (DE; $\mathrm{cm})$, número de hileras $(\mathrm{NH})$, número de granos por hilera (NGH), número total de granos (NG) del elote, peso de 200 granos frescos (PF200; g). Además se midieron características de calidad elotera, como contenido de sólidos solubles totales (SST; ${ }^{\circ}$ Brix); esta variable se midió en una muestra de $25 \mathrm{~g}$ de granos crudos a los que se les agregó $25 \mathrm{~mL}$ de agua destilada, se licuaron y con un refractómetro se midieron en ${ }^{\circ}$ Brix.

De los elotes restantes, tres de ellos se cocieron el día del corte a los que se calificó el sabor del grano (SAB; escala 1 a 5 , donde 5 fue para mejor sabor y 1 para el peor), por tres personas por separado. Los dos elotes 
restantes se utilizaron para calificar vida de anaquel (VA; misma escala que SAB) después de $5 \mathrm{~d}$ de cosechados. En planta se registraron los días a floración femenina (DF; días después de siembra).

Debido a que no hubo efecto de interacción con fechas de siembra, se decidió hacer un solo análisis combinado con las cuatro repeticiones que integraron los dos experimentos (dos fechas). Los datos fueron primero analizados como látice simple por separado; sin embargo, en todas las variables la eficiencia del diseño látice fue menor a 5 $\%$, comparado con el de bloques completos al azar, y no hubo necesidad de usar medias ajustadas por el análisis del látice. Luego, las medias de las variables que fueron significativas se analizaron como diseño de apareamiento dialélico mediante el programa DIALLEL (Burrow y Coors, 1994), programa que usa el modelo 1 de cruzamiento dialélico sugerido por Griffing (1956).

\section{RESULTADOS Y DISCUSIÓN}

Con el análisis de varianza (Cuadro 1) se detectó que las variables rendimiento (Rend), contenido de sólidos solubles totales (SST), número total de granos (NG), diámetro de elote $(\mathrm{DE})$, número de hileras $(\mathrm{NH})$, peso de 200 granos en elote (PF200), vida de anaquel (VA) y días a floración (DF) mostraron diferencias $(\mathrm{P} \leq 0.05)$ para el factor tratamientos o cruzas; en cambio, en las variables número de granos por hilera (NGH) y longitud de elote (LE) no hubo diferencias $(P \leq 0.05)$. Las variables significativas permitieron particionar la suma de cuadrados de los tratamientos de acuerdo con el modelo 1 de Griffing (1956), y así se encontró que todas las variables, excepto rendimiento, fueron significativas para la aptitud combinatoria general (ACG); en cambio, para la aptitud combinatoria específica (ACE), solo fueron significativas $(\mathrm{P} \leq 0.05)$ las variables Rend, SST, VA y DF. Las variables no significativas para la ACE, fueron NG, DE, NH y PF200.

En general, la ACG resultó predominantemente mayor que la ACE en todas las variables, excepto para rendimiento, lo que indica que los efectos aditivos para esas variables, son más importantes que los efectos no aditivos. Esta predominancia también ha sido encontrada en otros tipos de maíz y otras características (Hallauer y Miranda, 1981; Lamkey y Hallauer, 1984; Betrán et al., 2003; Doerksen et al., 2003). La mayor magnitud de los efectos aditivos indica la conveniencia de utilizar esquemas de selección intra-poblacional, como la selección masal, si el carácter fuera de alta heredabilidad; de lo contrario, convendría aplicar selección recurrente con progenies autofecundadas $S_{1}$ o $S_{2}$ donde el progreso a la selección sería más efectivo (Fehr, 1987).

Es decir, para las características eloteras de sólidos solubles, tamaño del elote ( $\mathrm{NG}, \mathrm{DE}$ y $\mathrm{NH}$ ), peso del grano (PF200) y vida de anaquel, así como para los días a floración femenina, los métodos de selección arriba mencionados serían más efectivos para explotar los efectos aditivos; en cambio, para el rendimiento de elote cuyos efectos no aditivos fueron más importantes, un método más adecuado para mejorar el rendimiento de maíz elotero es la explotación de la heterosis, ya sea a través de selección recurrente recíproca o del desarrollo de híbridos. La mayor magnitud de los efectos no aditivos indica que los efectos genéticos responsables del rendimiento en maíz elotero tienen una acción génica de sobredominancia o epistática dominante.

Cuadro 1. Cuadrados medios de ocho características con diferencias significativas, para seis maíces eloteros y sus cruzas dialélicas, directas y recíprocas. Xalisco, Nay. PV-2007.

\begin{tabular}{|c|c|c|c|c|c|c|c|c|c|}
\hline \multirow[b]{2}{*}{ Fuente de variación } & \multirow[b]{2}{*}{$\mathrm{gl}$} & \multicolumn{8}{|c|}{ Cuadrados medios } \\
\hline & & $\begin{array}{l}\text { REND } \\
\left(\mathrm{kg} \mathrm{ha}^{-1}\right)\end{array}$ & $\begin{array}{c}\text { SST } \\
\left({ }^{\circ} \text { Brix }\right)\end{array}$ & $\begin{array}{c}\text { NG } \\
\text { (número) }\end{array}$ & $\begin{array}{c}\mathrm{DE} \\
(\mathrm{cm})\end{array}$ & $\begin{array}{c}\mathrm{NH} \\
\text { (número) }\end{array}$ & $\begin{array}{l}\text { PF200 } \\
(\mathrm{g})\end{array}$ & $\begin{array}{c}\text { VA } \\
\text { (calificación) }\end{array}$ & $\begin{array}{c}\mathrm{DF} \\
\text { (días) }\end{array}$ \\
\hline Repeticiones & 3 & 20149380 & 5.34 & 31566 & 2.83 & 4.29 & 151 & 0.50 & 178 \\
\hline Cruzas & 35 & 1146508 & 0.24 & 7103 & 0.47 & 4.91 & 419 & 1.47 & 19 \\
\hline ACG & 5 & 933516 & 0.68 & 18339 & 1.76 & 19.36 & 1754 & 3.78 & 77 \\
\hline $\mathrm{ACE}$ & 15 & 1397127 & 0.18 & 5204 & 0.23 & 2.04 & 184 & 0.81 & 14 \\
\hline Recíprocas & 15 & 966886 & 0.50 & 5258 & 0.29 & 2.95 & 209 & 1.36 & 5 \\
\hline Error & 105 & 448420 & 0.07 & 3838 & 0.21 & 1.29 & 117 & 0.29 & 4 \\
\hline Total & 143 & & & & & & & & \\
\hline $\mathbf{R}^{2}$ & & 0.68 & 0.78 & 0.46 & 0.53 & 0.58 & 0.55 & 0.64 & 0.72 \\
\hline CV (\%) & & 14.8 & 14.4 & 16.4 & 7.9 & 9.1 & 11.5 & 9.9 & 3.0 \\
\hline
\end{tabular}

Rend = rendimiento de de elote; SST = sólidos solubles totales; NG = número de granos; DE = diámetro de elote; $\mathrm{NH}=$ número de hileras; PF200 = peso 200 granos de elote; VA = vida de anaquel; DF = días a floración femenina; gl = grados de libertad 
Los efectos recíprocos resultaron significativos $(\mathrm{P} \leq$ 0.05) para SST, NH, VA, Rend y PF200; pero no lo fueron para NG, DE y DF. Los efectos recíprocos se relacionan con los efectos maternos, los cuales han sido generalmente insignificantes en el rendimiento de grano del maíz común (Hallauer y Miranda, 1981). Pero en el rendimiento de elotes, cosechados 15 a 18 días después floración, los efectos maternos fueron importantes en esta investigación, al igual que para sólidos solubles, numero de hileras, vida de anaquel y del grano fresco.

En el rendimiento de elote (Rend), los efectos genéticos de aptitud combinatoria fueron favorables al criollo Jala (Cuadro 2). Por su efecto de ACG, las mejores cruzas fueron las del criollo Jala con un efecto positivo ( $\mathrm{P} \leq$ $0.01)$ de 955. Las cruzas del criollo Jala con las poblaciones Morado y Mecatán, tuvieron una ACE positiva, y además el criollo Jala fue también sobresaliente en rendimiento de elote $\left(\mathrm{kg} \mathrm{ha}^{-1}\right)$, según el promedio de las cruzas en que participó como progenitor, con un rendimiento de $12041 \mathrm{~kg} \mathrm{ha}^{-1}$. Los maíces criollos estudiados tienen reconocida adaptación específica a una localidad y no han sido mejorados, contrario a los híbridos comerciales que han sido mejorados y son ampliamente usados en diversos ambientes (Barrera et al., 2005).

Estos resultados indican que el criollo Jala es un progenitor apropiado para desarrollar maíces específicos para producción de elote, mediante el aprovechamiento de los efectos aditivos; también convendría recombinarlo con el híbrido comercial 'A7573' para mejorar características importantes de adaptación y tipo de planta. Si fuera de interés el desarrollo de híbridos, convendría utilizar un tipo de patrón heterótico entre los maíces Jala y Mecatán, cuyo cruzamiento mostró alta ACE, y por ello éstos son los progenitores adecuados para el aprovechamiento de efectos no aditivos. No obstante, de acuerdo con Barrera et al. (2005), algunas características agronómicas desfavorables reconocidas en los maíces criollos podrían llevar a retrocruzarlos con híbridos comerciales para propiciar mejores características de planta, procurando a la vez conservar los aspectos de calidad elotera.

Como característica de calidad, el contenido de sólidos solubles totales fue importante para el maíz Jala que tuvo el valor más alto $\left(2.53{ }^{\circ}\right.$ Brix $)$, seguido de los maíces Morado y 'D880' (Cuadro 3). En ACG, el maíz Jala tuvo efecto positivo y significativo (0.199), mayor que el resto de genotipos; de hecho, el híbrido 'A7573', extensivamente usado en México como elotero, y el maíz Mecatán tuvieron efectos negativos y significativos. Dada la importancia de los sólidos solubles como componente de calidad, genéticamente se pueden aprovechar los efectos aditivos significativos mostrados por el maíz Jala para el desarrollo de variedades mejoradas de polinización libre; en cambio, no hubo información clara para decidir sobre cruzamientos que aprovechen los respectivos efectos no aditivos en el desarrollo de híbridos de maíz elotero.

Los efectos de ACG para número de granos, el mejor efecto lo obtuvo el híbrido 'A7573' con un valor de 21.4, mientras que la mejor ACE la presentó la cruza Morado x Jala. No se detectó un efecto significativo positivo en las cruzas recíprocas. De acuerdo con los resultados obtenidos, el híbrido 'A7573' aprovecha mejor los efectos aditivos para la selección de variedades de polinización libre con alto número de granos en el elote, mientras que los criollos Morado y Jala mostraron los mejores efectos no aditivos apropiados para el desarrollo de híbridos. De acuerdo con resultados obtenidos (datos no mostrados), el germoplasma más adecuado para aprovechar los efectos aditivos son los maíces eloteros Jala y 'D880', para así lograr mayor diámetro de elote. Similarmente, los maíces 'A7573' y 'D880' son los más adecuados para la selección de maíces con mayor número de hileras, seguidos de Jala, Morado y Mecatán; los tres criollos, son los mejores para el peso de grano (PF200); Jala es el mejor para vida de anaquel; y 'A7573' el mejor para seleccionar por precocidad.

Cuadro 2. Rendimiento per se de progenitores (diagonal, $\mathrm{kg} \mathrm{ha}^{-1}$ ), efectos de aptitud combinatoria específica (arriba diagonal), efectos recíprocos (abajo diagonal), y efectos de aptitud combinatoria general $\left(\hat{g}_{i}\right)$. Xalisco, Nay. PV-2007.

\begin{tabular}{|c|c|c|c|c|c|c|c|c|}
\hline $\mathrm{p}$ & Progenitor & Morado & Mecatán & 'B810' & 'D880' & 'A7573' & Jala & Promedio de cruzas \\
\hline 1 & Morado & $9140^{\S}$ & $-583^{\dagger}$ & 654 & 70 & 118 & $1130 *$ & $10832(5)^{8}$ \\
\hline 2 & Mecatán & $215^{\ddagger}$ & 10863 & -485 & -114 & 182 & $1198 *$ & $11099(2)$ \\
\hline 3 & 'B810' & 650 & -100 & 11076 & $-1007 *$ & 187 & 260 & $10886(4)$ \\
\hline 4 & 'D880' & 251 & 189 & -810 & 10848 & -435 & 723 & $10586(6)$ \\
\hline 5 & 'A7573' & 848 & 85 & -551 & -1385 & 10300 & 707 & $11073(3)$ \\
\hline \multirow[t]{2}{*}{6} & Jala & 131 & -649 & 911 & -1026 & 196 & 8977 & $12041(1)$ \\
\hline & $\hat{g}_{i}$ & $-254^{\pi}$ & 12.7 & -200 & $-500 *$ & -13.2 & $955 * *$ & \\
\hline
\end{tabular}

* y ** Significancia a $\mathrm{P} \leq 0.05$ y $\mathrm{P} \leq 0.01$, respectivamente.

†Error estándar para efectos de la $\mathrm{ACE}=463$; "Error estándar para efectos recíprocos $=770$; "Error estándar para efectos de la ACG $=203$;

${ }^{\&}$ Número en paréntesis se refiere al orden de la cruza promedio; ${ }^{\S}$ Tukey $(0.05)=2159$. 
Cuadro 3. Contenido de sólidos solubles totales para progenitores per se (diagonal, grados brix), pero efectos de aptitud combinatoria específica (arriba diagonal), para efectos recíprocos (abajo diagonal), y para efectos de aptitud combinatoria general ( $\left.\hat{g}_{i}\right)$. Xalisco, Nay. PV-2007.

\begin{tabular}{|c|c|c|c|c|c|c|c|c|}
\hline $\mathrm{p}$ & Progenitor & Morado & Mecatán & 'B810' & 'D880' & 'A7573' & Jala & Promedio de cruzas \\
\hline 1 & Morado & $2.000^{\S}$ & $-0.153^{\dagger *}$ & 0.001 & 0.045 & 0.056 & $-0.261 * *$ & $1.71(4)^{\&}$ \\
\hline 2 & Mecatán & $-0.075^{\ddagger}$ & 1.550 & 0.141 & 0.064 & 0.024 & $-0.167 *$ & $1.59(6)$ \\
\hline 3 & 'B810’' & 0.175 & 0.088 & 1.780 & $-0.161 *$ & -0.013 & 0.033 & $1.76(3)$ \\
\hline 4 & ‘D880’ & $0.213 *$ & 0.138 & -0.075 & 1.850 & -0.103 & 0.093 & $1.76(2)$ \\
\hline 5 & 'A7573' & 0.188 & 0.063 & -0.163 & 0.050 & 1.650 & -0.097 & $1.62(5)$ \\
\hline \multirow[t]{2}{*}{6} & Jala & -0.225 & -0.075 & -0.038 & 0.175 & -0.100 & $2.530 *$ & $1.93(1)$ \\
\hline & $\left(\hat{g}_{i}\right)$ & $-0.016^{\pi}$ & -0.135 & 0.028 & 0.030 & $-0.106^{*}$ & $0.199 * *$ & \\
\hline
\end{tabular}

$* \mathrm{y} * *$ significancia $\mathrm{P} \leq 0.05 \mathrm{y} \mathrm{P} \leq 0.01$, respectivamente.

${ }^{\dagger}$ Error estándar para los efectos de la aptitud combinatoria específica $=0.0774$; "Error estándar para los efectos recíprocos $=0.1000$; "Error estándar para los efectos de la aptitud combinatoria general $=0.0340 ;{ }^{8}$ Número en paréntesis se refiere al orden de la cruza promedio; ${ }^{\S} \mathrm{Tukey}$ $0.05=0.696$.

\section{CONCLUSIONES}

Los efectos de aptitud combinatoria general fueron más importantes que los efectos de aptitud combinatoria específica en todas las características en los maíces eloteros estudiados, excepto para rendimiento de elote. Esto implica que los efectos aditivos fueron más importantes que los efectos de dominancia, y que es posible desarrollar variedades mejoradas especializadas como maíces eloteros.

El maíz criollo de la raza Jala mostró efectos aditivos importantes para producción de elote, en cuanto a sólidos solubles totales, diámetro de elote, tamaño de grano de elote y vida de anaquel. Es entonces una fuente germoplásmica importante para la formación de variedades mejoradas de polinización libre.

El maíz Jala mostró efectos no aditivos importantes en cruzamientos con el híbrido 'D880' y con los criollos Mecatán y Morado, para mejorar características diversas características agronómicas, por lo que posee también potencial como fuente importante en el desarrollo de híbridos.

\section{BIBLIOGRAFÍA}

Aguilar-Castillo J A, A Carballo-Carballo, F Castillo-González, A Santacruz-Varela, J A Mejía-Contreras, J Crossa-Hiriartte, G Baca-Castillo (2006) Diversidad fenotípica y variantes distintivas de la raza Jala de maíz. Agric. Téc. Méx. 32:57-66.

Barrera-Gutiérrez E, A Muñoz-Orozco, F Márquez-Sánchez, F Martínez-Garza (2005) Aptitud combinatoria en razas de maíz mejoradas por retrocruza limitada. I: Caracteres agronómicos. Rev. Fitotec. Mex. 28:231-242.

Betrán F J, J M Ribaut, D Beck, D Gonzalez de León (2003) Genetic diversity, specific combining ability, and heterosis in tropical maize under stress and nonstress environments. Crop Sci. 43:797-806.
Burrow M D, J G Coors (1994) DIALLEL: a microcomputer program for the simulation and analysis of diallel crosses. Agron. J. $86: 154-158$.

Doerksen T K, L W Kannenberg, E A Lee (2003) Effect of recurrent selection on combining ability in maize breeding populations. Crop Sci. 43:1652-1658.

Fehr W R (1987) Principles of Cultivar Development. Volume 1. Theory and Technique. Macmillan Publishing Company. New York, USA. 536 p.

Griffing B (1956) Concept of general and specific combining ability in relation to diallel crossing systems. Aust. J. Biol. Sci. 9:463493.

Hallauer A R, J B Miranda Fo (1981) Quantitative Genetics in Maize Breeding. Iowa State Univ. Press, Ames, IA, USA. 468 p.

Lamkey K R, A R Hallauer (1984) Comparison of maize populations improved by recurrent selection. Maydica 29:357-374.

Rice E B, M E Smith, S E Mitchell, S Kresovich (2006) Conservation and change: a comparison of in situ and ex situ conservation of Jala maize germplasm. Crop Sci. 46:428-436.

Vidal-Martínez V A (1993) Guía para Cultivar Maíz Elotero de Riego en Nayarit. Folleto para productores No. 5, Campo Experimental Santiago Ixcuintla, INIFAP, Santiago Ixcuintla, Nayarit. 10 p. 